THE Astrophysical Journal, 354:389-399, 1990 May 10

(C) 1990. The American Astronomical Society. All rights reserved. Printed in U.S.A.

\title{
A MODEL FOR THE DISTRIBUTION OF THE INTERGALACTIC MEDIUM
}

\author{
DONGSU RYU \\ Department of Astronomy, University of Texas; and NASA/Fermilab Astrophysics Center, Fermilab \\ ETHAN T. VISHNIAC ${ }^{1}$ \\ Department of Astronomy, University of Texas \\ AND \\ Wei-Hwan Chiang \\ Department of Astronomy, University of Texas; and IBM Corporation \\ Received 1988 December 5 ; accepted 1989 November 15
}

\begin{abstract}
We have investigated the evolution and distribution of the intergalactic medium in a universe dominated by cold dark matter. The Einstein-de Sitter universe with $\Omega_{0}=1$ and $h=0.5$ has been considered (here $h=$ $H_{0} / 100 \mathrm{~km} \mathrm{~s}^{-1} \mathrm{Mpc}^{-1}$ and $H_{0}$ is the present value of the Hubble constant). We have assumed that initially dark matter composes $90 \%$ and baryonic matter composes $10 \%$ of the total mass. During the model calculations, we have identified galaxies, which in this context mean any objects with a significant energy feedback into the IGM, following some simple criteria, and deposited $10^{60}$ ergs of energy into the IGM around the galaxies over a period of $10^{8}$ yr. Calculations have been performed with $32^{3}$ dark matter particles and $32^{3}$ cells in a cube with comoving side length $L=9.6 h^{-1} \mathrm{Mpc}$.

In our model, galaxies form and eject energy into the IGM from $z \sim 20$ up to present. The distribution of the IGM is dominated by large connected structures. The power spectrum and two-point correlation function of the IGM show a suppressed growth due to the energy injected from galaxies and the mass subtraction to form galaxies. The high-temperature regions of the IGM correspond to the low-density regions and the lowtemperature regions correspond to the high-density regions. The temperature of the IGM increases from $z=1$ to $z=0$, while the pressure decreases. The present temperature distribution shows a peak at $\sim 10^{7} \mathrm{~K}$. The mass fraction of the IGM with the temperature below $10^{5} \mathrm{~K}$ is negligible, indicating almost all the hydrogen is ionized. Pressure variation is smaller than the density and temperature variations, even though pressure equilibrium is not achieved. Temperature fluctuations in the cosmic microwave background radiation due to the scattering electrons in the hot IGM gas create temperature anisotropies which are smaller than the observational limit. The amount of X-ray radiation emitted by the hot IGM plasma is also expected to be much smaller than that observed in the X-ray background.
\end{abstract}

Subject headings: cosmology - dark matter — early universe — galaxies: clustering — galaxies: formation — galaxies: intergalactic medium

\section{INTRODUCTION}

Among the models which have proposed to explain the present structure of our universe, a universe dominated by cold dark matter (CDM) with biased galaxy formation has been the most successful one (Blumenthal et al. 1984; Davis et al. 1985; Bardeen et al. 1986; White et al. 1987; Frenk et al. 1988). In such a universe, there is a substantial amount of baryonic matter, in addition to the dark matter, and those baryons reside inside galaxies or in the intergalactic medium (IGM). The baryonic matter density in units of the critical density, $\Omega_{\mathrm{BM}}$, is limited by the theory of big bang nucleosynthesis and its upper bound is placed to be $\sim 0.1$ (Yang et al. 1984; KurkiSuonio et al. 1989). The most direct evidence of the existence of the IGM comes from the Ly $\alpha$ absorption lines in high redshift quasar spectra. These were first identified by Lynds (1971) and subsequently studied in great detail by Sargent et al. (1980). These lines are generally interpreted as being due to intervening gas coulds of almost pure $\mathrm{H}$ and $\mathrm{He}$ distributed randomly in space. The analysis of Sargent et al. showed that the clouds are pressure bounded with hydrogen number densities from $10^{-4}$ to $4 \times 10^{-3} \mathrm{~cm}^{-3}$, diameters from $10^{20}$ to $10^{23} \mathrm{~cm}$,

\footnotetext{
${ }^{1}$ Presidential Young Investigator and Alfred P. Sloan Fellow.
}

and temperatures $\sim 3 \times 10^{4} \mathrm{~K}$, and are embedded in a relatively tenuous surrounding gas with hydrogen number density from $10^{-5}$ to $10^{-4} \mathrm{~cm}^{-3}$ and temperature from $3 \times 10^{5} \mathrm{~K}$ to $10^{7} \mathrm{~K}$. The clouds appear to be distributed in space much more uniformly and clustered much less than what we would expect from the galaxy distribution (Ostriker, Bajtlik, and Duncan 1988; Crotts 1989). The Gunn-Peterson test constrains most of the hydrogen in the $\mathrm{L}_{\alpha}$ clouds and surrounding gas to be highly ionized (Gunn and Peterson 1965).

Until now, most studies on the CDM universe have considered only the distribution of the dark matter and compared that with the observed distribution of galaxies. However, recently there have been several papers, including those by Carlberg (1988) and Chiang, Ryu, and Vishniac (1989), where baryons have been included in a very approximate way. In our previous paper (Chiang, Ryu, and Vishniac 1989), although we studied the evolution of the IGM, we did not follow the evolution of the dark matter explicitly. Instead, we calculated the gravitational force using the initial gravitational potential. In addition, although we identified objects which subsequently released energy back to the IGM, we did not subtract object masses from the IGM and did not follow the motion of the objects. 
In this paper, we have relaxed some of the assumptions used in our previous paper. For example, we have followed the evolution of the dark matter, as well as the evolution of the IGM. Also, some of the baryonic matter has been subtracted when objects form, and the motion of the objects formed has been followed explicitly. However, the specific question addressed by this paper remains the same as that of the previous paper: how would the IGM left over from galaxy formation evolve if there is significant energy feedback from the pregalactic objects? In $\S$ II, we describe the method we have used to solve this problem. In our calculations, the Einstein-de Sitter universe with $\Omega_{0}=1$ has been assumed and we have taken $h=0.5$. In $\S$ III, we present the results of our calculations, mainly the properties of the IGM. Finally, the summary and conclusions of our work are given in $\S$ IV.

\section{THE MODELS}

\section{a) Basic Equations}

We describe the evolution and distribution of the IGM in a universe dominated by CDM, on length scales small compared to the radius of the universe so that the Newtonian approximation is valid. Since the dark matter determines the overall dynamics of structures in such a universe, we follow the evolution of the dark matter, as well as the evolution of the IGM. Our model universe initially contains two components-dark matter and baryonic matter. The dark matter is assumed to comprise $90 \%$ of the total mass of the universe $\left(\Omega_{\mathrm{DM}}=0.9\right)$, while the baryonic matter accounts for the other $10 \%\left(\Omega_{\mathrm{BM}}=\right.$ 0.1 ). The baryonic matter consists of $\mathrm{H}$ and $\mathrm{He}$, with the abundance of $\mathrm{He}$ equal to $10 \%$ of that of $\mathrm{H}$ by number. Later, some of the baryonic matter ( $\sim 7 \%$ by mass, on average) is subtracted, in order to form objects which subsequently release energy back into the remaining baryonic matter, or the IGM, Sometimes, we will refer to the objects as galaxies for convenience. However, the word galaxy should be interpreted broadly as any object formed that ejects a significant amount of energy into the IGM.

Following Chiang, Ryu, and Vishniac (1989), we define a set of dimensionless variables in the comoving coordinates as follows:

$$
\begin{aligned}
& \tilde{t}=\frac{t}{t_{0}}, \\
& \tilde{x}_{i}=\frac{x_{i}}{a x_{0}}, \\
& \tilde{v}_{i}=\frac{v_{i}}{a x_{0} / t_{0}}, \\
& \tilde{\rho}=\frac{\rho}{\rho_{0}}, \\
& \tilde{e}=\frac{e}{a^{2} \rho_{0} x_{0}^{2} / t_{0}^{2}}, \\
& \tilde{\phi}=\frac{\phi}{a^{2} x_{0}^{2} / t_{0}^{2}},
\end{aligned}
$$

where $a$ is the expansion parameter, $t$ is the time, $x_{i}$ are the proper coordinates, $v_{i}$ is the peculiar velocity, $\rho$ is the mass density, $e$ is the internal energy per unit volume, and $\phi$ is the peculiar gravitational potential. Here, we choose three basic units of normalization, that is, length $x_{0}$, time $t_{0}$, and density $\rho_{0}$. In our calculations, we fix $x_{0}$ as the size of comoving cell, $t_{0}$ as the present age of the universe, and $\rho_{0}$ as the average density of the universe:

$$
\begin{aligned}
& x_{0}=\frac{L}{n}, \\
& t_{0}=\frac{2}{3} \frac{1}{H_{0}}, \\
& \rho_{0}=\frac{3 H^{2}}{8 \pi G},
\end{aligned}
$$

where $L$ is the comoving side length of the total computational box, $n$ is the number of grids in one direction, $H=\dot{a} / a$ is the Hubble constant, and $H_{0}$ is the present value of the Hubble constant.

Then, the dark matter is described by the folowing Newton's equations of motion

$$
\begin{gathered}
\frac{d \tilde{x}_{i \mathrm{DM}}}{d t}=\tilde{v}_{i \mathrm{DM}}, \\
\frac{d \tilde{v}_{i \mathrm{DM}}}{d t}+\frac{4}{3} \frac{\tilde{v}_{i \mathrm{DM}}}{\tilde{t}}=-\tilde{\nabla}_{i} \tilde{\phi},
\end{gathered}
$$

while the IGM is described by the following hydrodynamical equations

$$
\begin{gathered}
\frac{\partial \tilde{\rho}_{\mathrm{IGM}}}{\partial \tilde{t}}+\tilde{\nabla}_{j}\left(\tilde{\rho}_{i \mathrm{GM}} \tilde{v}_{j \mathrm{IGN}}\right)=0, \\
\frac{\partial \tilde{M}_{i}}{\partial t}+\tilde{\nabla}_{j}\left(\tilde{M}_{i} \tilde{v}_{j \mathrm{IGM}}\right)+\frac{4}{3} \frac{\tilde{M}_{i}}{\tilde{t}}+\tilde{\rho}_{\mathrm{IGM}} \tilde{\nabla}_{i} \tilde{\phi}+\tilde{\nabla}_{i} \tilde{p}=0, \\
\frac{\partial \tilde{E}}{\partial \tilde{t}}+\tilde{\nabla}_{j}\left[(\tilde{E}+\tilde{p}) \tilde{v}_{j \mathrm{IGM}}\right]+ \\
+\frac{8}{3} \frac{\tilde{E}}{\tilde{t}}-\frac{4}{3} \frac{\tilde{e}}{\tilde{t}}+2 \frac{\tilde{p}}{\tilde{t}} \\
+\tilde{\rho}_{\mathrm{IGM}} \tilde{v}_{j \mathrm{IGM}} \tilde{\nabla}_{j} \tilde{\phi}=\tilde{\Gamma}-\tilde{\Lambda},
\end{gathered}
$$

where $\tilde{M}_{i}$ is the peculiar momentum of the IGM, $\tilde{p}$ is the pressure, $\widetilde{E}$ is the peculiar total energy per unit volume, $\Gamma$ is the heating rate, and $\Lambda$ is the cooling rate. The combined peculiar gravitational potential of the dark matter, galaxies, and IGM is then given by Poisson's equation as

$$
\tilde{\nabla}^{2} \tilde{\phi}=\frac{2}{3} \frac{1}{\tilde{t}^{2}}\left(\tilde{\rho}_{\mathrm{DM}}+\tilde{\rho}_{g}+\tilde{\rho}_{\mathrm{IGM}}-1\right)
$$

where $\tilde{\rho}_{\mathrm{DM}}$ is the dark matter mass density and $\tilde{\rho}_{g}$ is the galaxy mass density.

\section{b) Initial Conditions}

We use initial conditions which describe constant curvature adiabatic perturbations in a universe dominated by CDM. Inflation and linear perturbation theory predicts a primordial power spectrum for constant curvature adiabatic perturbations at early times, produced during inflation, and the subsequent growth of the power spectrum in the CDM-dominated universe. We use the form given by Davis et al. (1985) as the initial power spectrum:

$$
P_{k}=\frac{A k}{\left(1+\alpha k+\beta k^{3 / 2}+\gamma k^{2}\right)^{2}},
$$

where $A$ is a normalization constant. If $k$ is expressed in terms 
of comoving length units, the parameters take the values $\alpha=1.7 u, \beta=9.0 u^{3 / 2}$, and $\gamma=1.0 u^{2}$ where $u=\left(\Omega_{0} h^{2} \theta^{-2}\right)^{-1}$ Mpc, $\Omega_{0}$ is the present value of the cosmological density parameter which is taken as $1, h$ is the present value of the Hubble constant in units of $100 \mathrm{~km} \mathrm{~s}^{-1} \mathrm{Mpc}^{-1}$ which is taken as 0.5 , and $\theta$ is the present temperature of the cosmic microwave background radiation in units of $2.7 \mathrm{~K}$ which is also taken as 1 .

The initial conditions for the dark matter were generated using the Zel'dovich approximation following the algorithm described by Efstathiou et al. (1985). Using the initial power spectrum, we obtained a realization of the random Gaussian density distribution by assigning random phases to each wave mode. At the same time we obtained the corresponding peculiar gravitational potential on the spatial grid. Starting from the center of grid cells, particles representing the dark matter were moved and assigned positions and peculiar velocities proportional to the gradient of the peculiar gravitational potential. This procedure was performed with $32^{3}$ dark matter particles and $32^{3}$ cells in a cube with comoving side length $L=9.6 h^{-1} \mathrm{Mpc}$ using periodic boundary conditions, and reproduced the desired power spectrum up to the Nyquist frequency, $k_{\mathrm{N}}=2 \pi 16 / L$. The initial conditions for the baryonic matter, or the IGM, were chosen to be compatible with those for the dark matter. The density of the IGM was calculated by assigning the dark matter particles to $32^{3}$ grid cells with the cloud in cell (CIC) scheme and dividing by 9 , and the peculiar velocities were derived from the gradient of the peculiar gravitational potential. In all cases, the peculiar velocites were set so that only the growing modes of density fluctuations were present. The IGM was assumed to have a negligible amount of internal energy with temperature $T=0$ at the beginning.

We begin our calculations when the normalized expansion parameter $\tilde{a}=a / a_{0}=0.01$ where $a_{0}$ is the present value of the expansion parameter, and integrate the equations of motion numerically up to the present universe with $\tilde{a}=1$. The amplitude of the initial perturbations was chosen so that the linear extrapolation of the initial mass fluctuation $(\langle\delta M / M\rangle)$, which is the same for both the dark matter and the baryonic matter in our model, to the present epoch agrees with the present mass fluctuation, which has been derived from the observed distribution of galaxies (see Peebles 1982), on a scale corresponding the size of three cells. In our calculations, the epoch can not be rescaled because cooling and heating processes depend on density, and therefore on the epoch. Compton cooling depends explicitly on the redshift $z$. This means some initial normalization for $\boldsymbol{P}_{\boldsymbol{k}}$ must be chosen beforehand. This is difficult since $\delta_{\text {galaxy }} \neq \delta_{\text {DM }}$. Hence, our procedure for determining the initial amplitude contains a substantial uncertainty.

Four sets of the initial conditions were set up corresponding to different realizations, and the results have been properly averaged over the four runs with the four initial conditions in order to reduce statistical fluctuations.

\section{c) The Numerical Procedure}

Newton's equations of motion for the dark matter particles were integrated numerically using a time centered method (described below), instead of the usual leap-frog method, since the time step should be controlled by the hydrodynamics of the IGM:

step 1 ,

$$
\begin{gathered}
\tilde{x}_{i n+1}^{\prime}=\tilde{x}_{i n}+\tilde{v}_{i n} \Delta t, \\
\tilde{v}_{i n+1}^{\prime}=\tilde{v}_{i n}-\frac{4}{3} \frac{\tilde{v}_{i n}}{\tilde{t}} \Delta \tilde{t}-\tilde{\nabla}_{i} \tilde{\phi}_{n}\left(\tilde{x}_{i n}\right) \Delta \tilde{t},
\end{gathered}
$$

step 2,

$$
\begin{aligned}
& \tilde{x}_{i n+1 / 2}^{\prime}=\frac{\tilde{x}_{i n}+\tilde{x}_{i n+1}^{\prime}}{2}, \\
& \tilde{v}_{i n+1 / 2}^{\prime}=\frac{\tilde{v}_{i n}+\tilde{v}_{i n+1}^{\prime}}{2},
\end{aligned}
$$

step 3,

$$
\begin{gathered}
\tilde{x}_{i n+1}=\tilde{x}_{i n}+\tilde{v}_{i n+1 / 2}^{\prime} \Delta \tilde{t}, \\
\tilde{v}_{i n+1}=\tilde{v}_{i n}-\frac{4}{3} \frac{\tilde{v}_{i n+1 / 2}^{\prime}}{\tilde{t}} \Delta \tilde{t}-\tilde{\nabla}_{i} \tilde{\phi}_{n+1 / 2}^{\prime}\left(\tilde{x}_{i n+1 / 2}^{\prime}\right) \Delta \tilde{t},
\end{gathered}
$$

where $\Delta t$ is the length of the time step and the subscript $n$ denotes the number of the time step.

The hydrodynamical conservation equations for the IGM were integrated using the flux corrected transport (FCT) method (Boris and Book 1973, 1976; Book, Boris, and Hain 1975) with the fully multidimensional flux limiting algorithm (Zalesak 1979). Since the FCT method was originally developed to handle shock problems, the numerical diffusion, needed to stabilize shock discontinuity, is expected to produce a substantial amount of smoothing, especially on small scales, in our problem where the mass density is initially distributed randomly from one cell to the next. The length of the time step was decided from the usual Courant condition and from the restriction connected with the cooling time scale.

The peculiar gravitational potential in the Poisson equation was calculated with the fast Fourier transformation (FFT) technique, where the dark matter and galaxy mass densities were derived from the CIC mass assignment scheme. The peculiar gravitational force on the IGM was obtained at the center of each mesh cell by differentiating the potential. The peculiar gravitational forces on the dark matter particles and galaxies were obtained using the particle mesh (PM) method (Hockney and Eastwood 1981), by interpolating the forces on the center of the adjacent eight cells in a way compatible with the CIC mass assignment scheme.

For the cooling term $\Lambda$ in the energy equation of the IGM, we have used the equilibrium cooling curve for a pure $\mathrm{H}$ and He primordial plasma given by Kang and Shapiro (1990), which includes recombination cooling, dielectric recombination cooling, ionization cooling, line cooling, bremsstrahlung cooling, and Compton cooling.

During our calculations, we have formed objects, or galaxies, from the IGM which release energy back into the IGM, if certain criteria have been satisfied. In a cell, a galaxy has formed in the center of the cell if

$$
\begin{aligned}
\rho_{t} & \geq v_{1} \bar{\rho}_{1}, \\
\rho_{\mathrm{IGM}} & \geq v_{2} \bar{\rho}_{2},
\end{aligned}
$$

where $\rho_{t}$ is the mass density of the dark matter and the IGM, $\rho_{\text {IGM }}$ is the mass density of the IGM, $\bar{\rho}_{1}$ is the average total mass density of the universe of the dark matter, IGM, and galaxies, and $\bar{\rho}_{2}$ is the average baryonic matter mass density of the universe of the IGM and galaxies. The first condition is necessary because the region of a density perturbation should break away from the general Hubble expansion to form a gravitationally bound object. The density of a spherical perturbation relative to the background density at maximum expansion is $9 \pi^{2} / 16=5.55$ (Peebles 1980). However, because of numerical diffusion on small scales present in calculating the gravitational force, we expect a smaller value of $v_{1}$. The second 
condition is introduced because in order to form a visibly luminous object, there should be a substantial amount of baryonic matter included. The value of $v_{2}$ may depend not only on the detailed process of the formation of the bound object but also on the amount of the numerical diffusion present in the fluid code we used. We have used 3.5 for $v_{1}$ and 2.5 for $v_{2}$ which give $\sim 815$ objects at the present epoch. This corresponds to one object per $1.1 h^{-3} \mathrm{Mpc}^{3}$ volume. However, our choices of $v_{1}$ and $v_{2}$ are somewhat arbitrary and there is room for further refinement.

Once a galaxy has formed in a cell, the mass corresponding to

$$
m_{g}=v_{3} \rho_{\mathrm{IGM}} V,
$$

where $V$ is the volume of the cell, has been subtracted from the IGM, in order to mimic the formation of the galaxy out of the IGM. Since it is difficult to believe that all the baryonic matter around the density perturbation is converted into a bound object, and also because of numerical difficulties, we have used 0.9 , instead of 1 , for $v_{3}$. However, the above mass subtracted from the IGM is completely controlled by the cell size we have chosen $\left(0.3 h^{-1} \mathrm{MPc}\right)$ and the density criteria for galaxy formation, so it should be considered as the mass accumulated in the galaxy-forming cell than the real galaxy mass. The velocity of the galaxy has been set to be equal to that of the IGM in the cell, and the subsequent motion of the galaxy has been followed using the Euler's first-order method.

After each object has formed, it releases $10^{60}$ ergs of energy into the IGM over a period of $10^{8} \mathrm{yr}$ on the adjacent eight cells in a way similar to the CIC mass assignment scheme. If the energy ejection is due to superwinds from early starbursts in galaxies, this corresponds to $10^{9}$ supernovae, each with $10^{51}$ ergs, per $10^{8} \mathrm{yr}$, or a supernova rate of $\sim 10$ per yr. Also $10^{60}$ ergs is approximately the amount of energy released by an individual quasar during its lifetime.

\section{RESULTS}

\section{a) Pattern of Galaxy Formation}

Figure 1 shows the rate of galaxy formation as a function of time. All the galaxies from the four runs, a total of 3266 galaxies, have been included. The galaxy formation rate determines the pattern of heating of the IGM from pregalactic objects. If we had employed different criteria for galaxy formation, different rates of galaxy formation are expected. For example, if we use only the criterion based on the density distribution of the IGM, equation (2.24), that is, galaxies form at the high-density regions of the IGM, regardless of the density distribution of the dark matter, all the galaxies form during a short period of time, between $z \sim 20$ and $z \sim 5$. Consequently, the resulting distribution of the IGM should be quite different from that of the present calculations, since it depends critically on its thermal history, including heating from the pregalactic objects (see Chiang, Ryu, and Vishniac 1989).

For each galaxy formed, a mass between $1.7 \times 10^{9} h^{-1} M_{\odot}$ and $7.7 \times 10^{9} h^{-1} M_{\odot}$ with an average of $2.1 \times 10^{9} h^{-1} M_{\odot}$ has been subtracted from the IGM. Since the spatial number density of the galaxies is $\sim 0.92$ per $1 h^{-3} \mathrm{Mpc}^{3}$, the total fraction of the baryonic matter which has been subtracted is $\sim 7 \%$. Again, as we mentioned in $\S$ II $c$, the above subtracted mass is controlled by the scheme of galaxy formation, not by real physical processes, in our calculations.

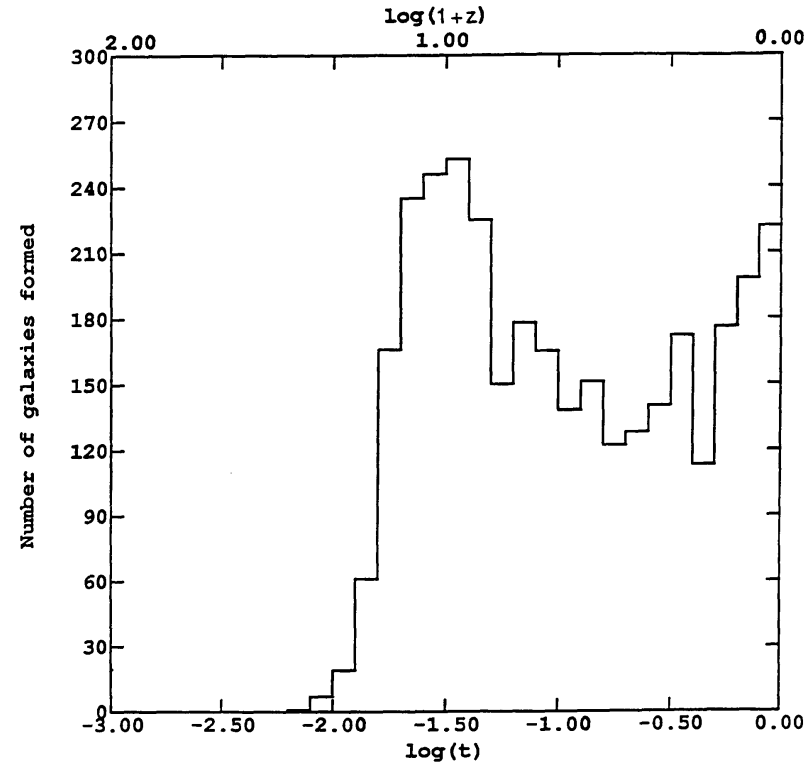

FIG. 1.-The number of galaxies formed as a function of time. The plot has been drawn with all the 3266 galaxies from the four runs.

\section{b) Morphology}

Figure 2 shows the three-dimensional density contour plots of the IGM at $z=1$ and $z=0$ from the $a$ run. Different runs with different realizations of the initial conditions are expected to show the same qualitative behavior. However, the overall topology associated with the density distribution depends on the density level used. Here, the surfaces correspond to a density level 2.5 times the mean density of the baryonic matter. In Figure 3, the two-dimensional density contour plots of the IGM at $z=1$ and $z=0$ from the same run have been drawn. The density has been calculated with the IGM mass located between $y=24$ and $y=32$. The solid lines indicate the regions with density higher than or equal to the mean density of the baryonic matter, and the dashed lines indicate those with density lower than the mean density. The interval of the contour lines corresponds to 0.3 times the mean density of the baryonic matter.

The main features in the above plots are the following: first, the distribution is dominated by large connected structures. Second, the volume contained in the high-density regions of the IGM shrinks a little bit in comoving space from $z=1$ to $z=0$, but does not change very much in real space. This second feature does not agree with that of our previous calculations (Chiang, Ryu, and Vishniac 1989). In the previous calculations, most of the galaxies formed and stopped heating the IGM before $z=1$; hence, the high-density regions shrink continuously due to gravitational forces between $z=1$ and $z=0$. On the other hand, in the present calculations, there are still a significant number of active galaxies heating the high-density regions of the IGM between $z=1$ and $z=0$ and the resulting pressure forces resist further condensation of the high-density regions.

\section{c) Power Spectrum and Two-Point Correlation Function}

We have calculated the density power spectrum, $P_{k}$, by Fourier transforming density fluctuations and binning to the nearest half integral wavenumbers. The comoving wavenumber is given in units such that $k=1$ corresponds to the funda- 


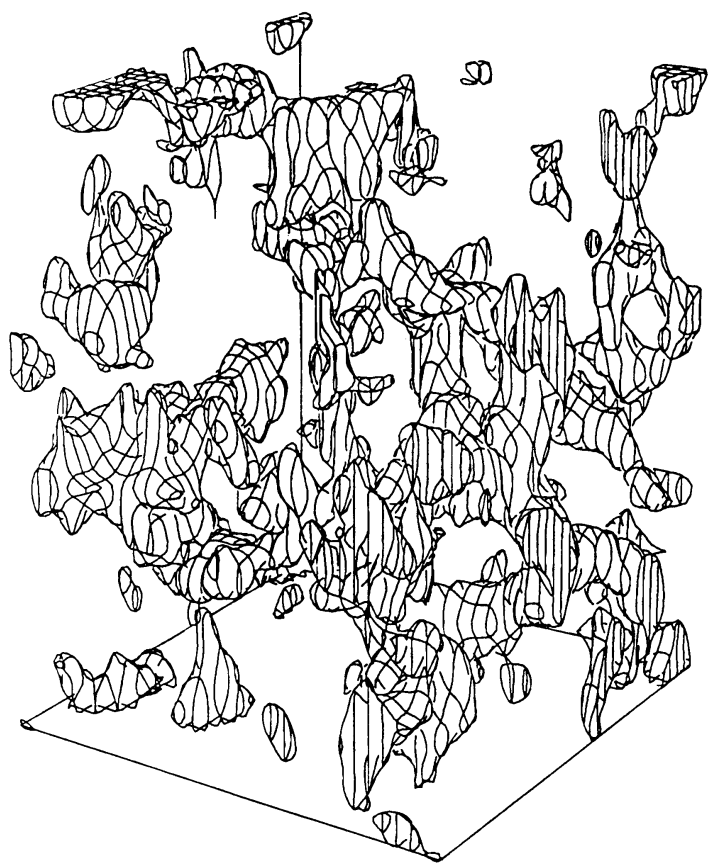

FIG. $2 a$

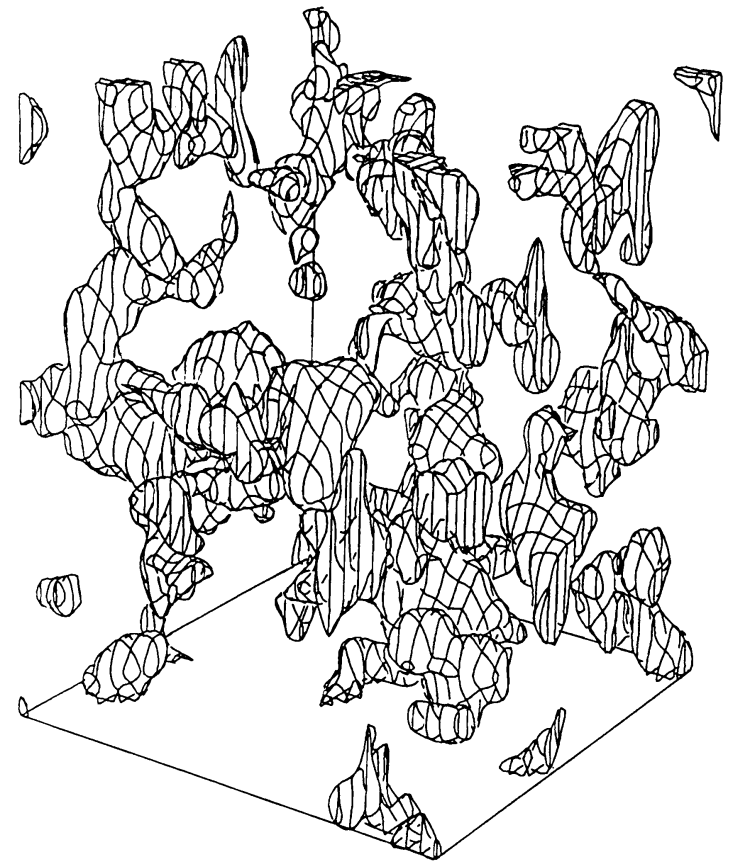

FIG. $2 b$

Fig. 2.-The three-dimensional density contour plots of the $\operatorname{IGM}(a)$ at $z=1$ and $(b)$ at $z=0$ from the $(a)$ run. The high-density regions with density higher than 2.5 times the mean density of the baryonic matter have been plotted.

mental wave of the computational box with comoving wavelength $\lambda=9.6 h^{-1} \mathrm{Mpc}$. The power spectra have been calculated up to the Nyquist frequency, $k_{\mathrm{N}}=16$ and averaged over the four runs. Figure $4 a$ shows the evolution of the power spectrum of the IGM. The late growth of the power of the IGM is greatly suppressed due to two factors: first, the pressure caused by the energy released from young galaxies works against gravity and is expected to suppress the growth of the power by smoothing out small-scale features, eliminating highfrequency power. Second, removing the IGM from the highdensity regions when galaxies form is expected to have a similar effect on the growth of the power at intermediate and low frequencies.

The two-point correlation function, $\xi(x)$, has been calculated

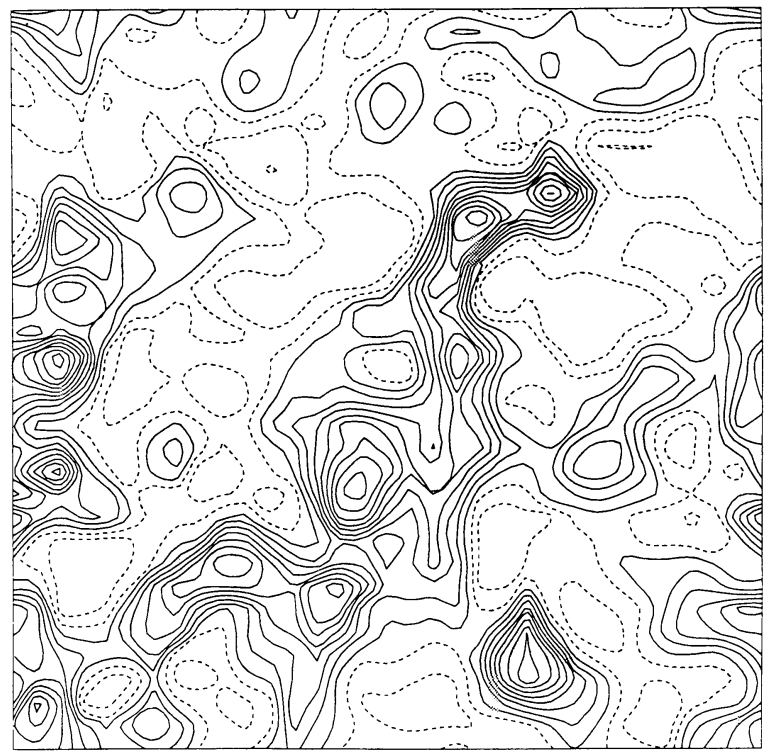

FIG. $3 a$

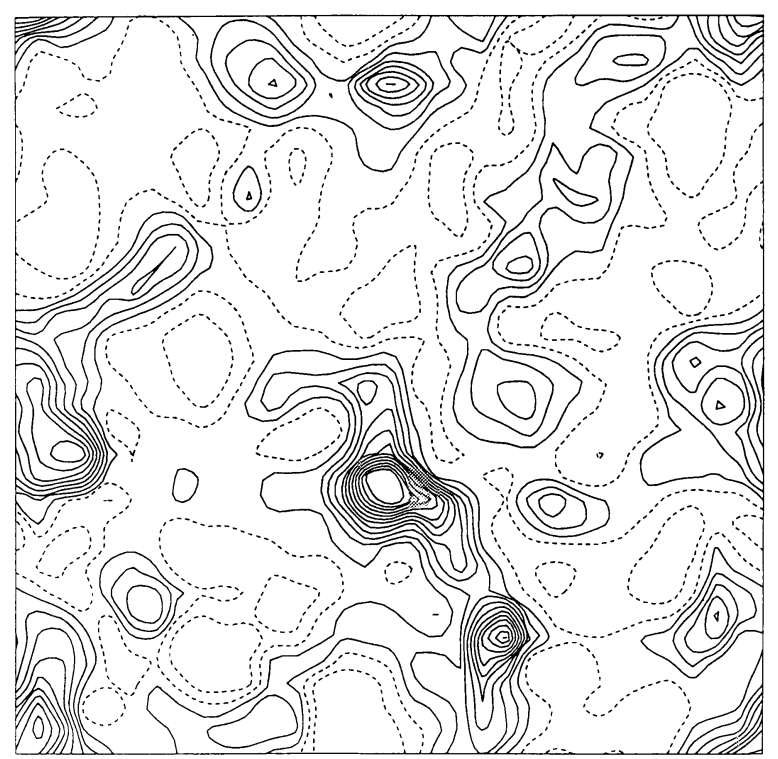

FIG. $3 b$

Fig. 3.-The two-dimensional density contour plots of the IGM $(a)$ at $z=1$ and $(b)$ at $z=0$ from the same run as that of Fig. 2. The density is the average density of the IGM, located between $y=24$ and $y=32$. The solid lines correspond to the high-density regions with density higher than or equal to the mean density of the baryonic matter, and the dashed lines correspond to the low-density regions with density lower than the mean density. The increment of the contour lines is 0.3 times the mean density. 


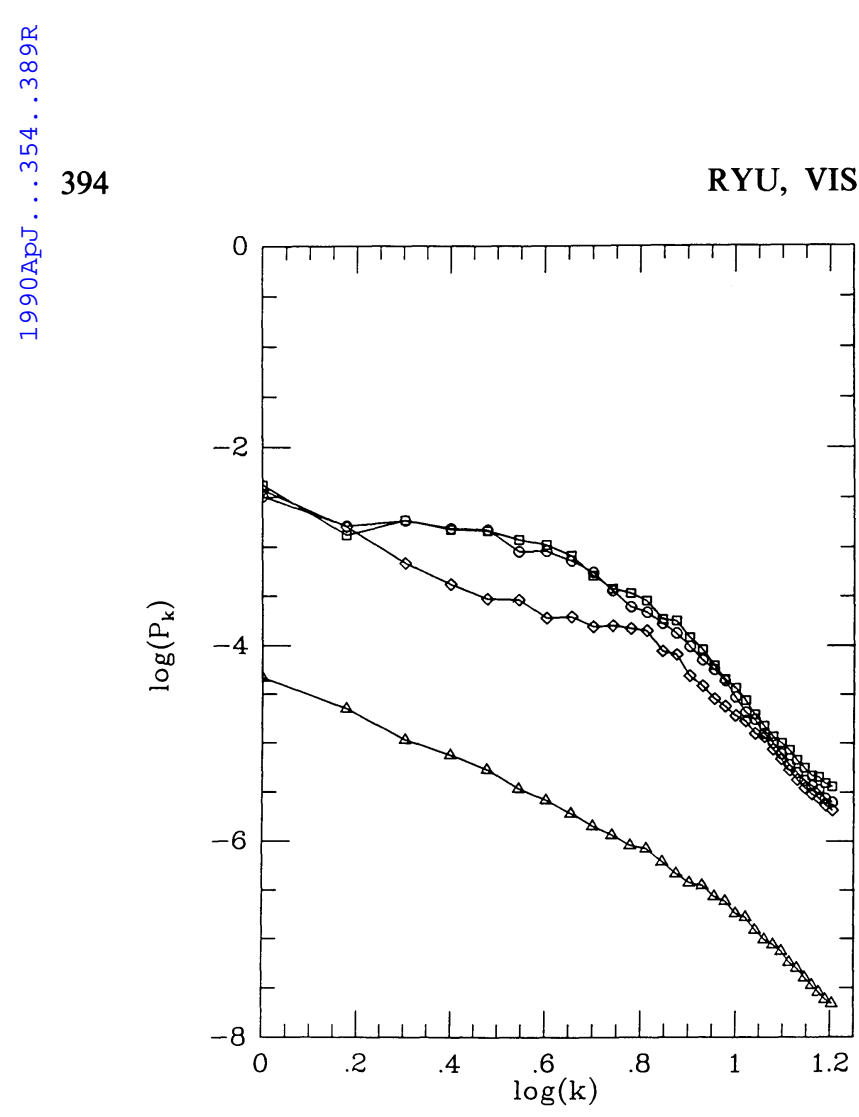

FIG. $4 a$

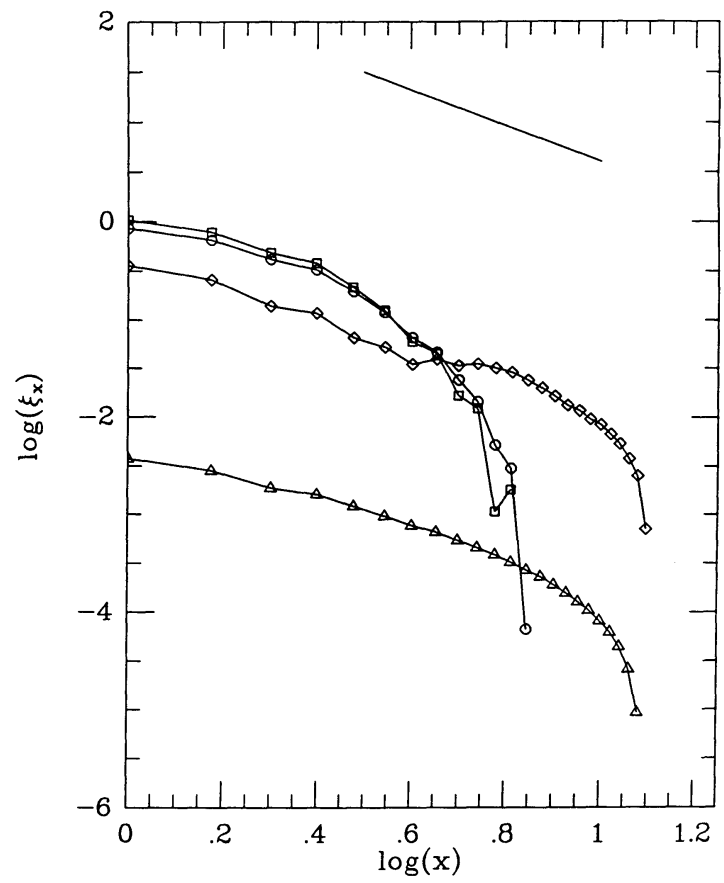

FIG. $4 b$

FIG. 4. - The evolution of $(a)$ the power spectrum and $(b)$ the two-point correlation function of the IGM. Lines correspond to $\tilde{a}=0.01$ (triangles), $\tilde{a}=0.1$ (diamonds), $\tilde{a}=0.5$ (squares), and $\tilde{a}=1.0$ (circles). The comoving wavenumber $k=1$ corresponds to the wavelength of the computational box size, and the separation $x$ is given in units of one cell size. Each plot has been averaged over the four runs. A line with a power-law index 1.8 which represents the observed galaxy-galaxy two-point correlation function is plotted for comparison in $(b)$.

by Fourier transforming the corresponding power spectra. Figure $4 b$ shows the evolution of the two-point correlation function of the IGM. Here, the comoving separation $x$ is given in units of one cell size, $0.3 h^{-1} \mathrm{Mpc}$, and the plots represent the average over the four runs. A line with a power-law index 1.8 which fits the observed galaxy-galaxy two-point correlation function is plotted for comparison. As expected from the power spectrum, the two-point correlation function also shows a substantial suppression in the late growth of amplitude and indicates the IGM should be less clustered than dark matter. This agrees qualitatively with the observations which indicate $\mathrm{L}_{\alpha}$ clouds are less clustered than galaxies (Ostriker et al. 1988; Crotts 1989).

In our calculations, since we are dealing with a small region of the universe (a $9.6^{3} h^{-3} \mathrm{Mpc}^{3}$ box), we expect our computational box to become saturated near the end of the calculations. Indeed, the power spectrum of dark matter indicates the growth of its fundamental mode is $\sim 40 \%$ below that expected from the linear theory. However, the overall evolution of the IGM, which is rather controlled by the hydrodynamics around the end of the calculations, is expected to suffer less from saturation than the dark matter.

\section{d) Temperature Distribution and Properties of the IGM}

In order to get a qualitative feeling for the overall temperature structure of the IGM, two-dimensional temperature contour plots at $z=1$ and $z=0$ have been shown in Figure 5 . The plots have been produced from the same run as that used to make the two-dimensional density contour plots of the IGM in Figure 3, and the temperature has been calculated from the internal energy of the IGM located between $y=24$ and $y=32$. In Figure $5 a$, the solid lines indicate regions with the temperature higher than or equal to $10^{7} \mathrm{~K}$, and the dashed lines indicate those with the temperature lower than $10^{7} \mathrm{~K}$. In Figure $5 b$, the solid lines correspond regions with the temperature higher than or equal to $2 \times 10^{7} \mathrm{~K}$ and the dashed lines correspond those with the temperature lower than $2 \times 10^{7} \mathrm{~K}$. The contour lines increase in a logarithmic scale with the increment $10^{0.1}$. If we compare the temperature contour plots with the density contour plots of the IGM, we can easily see that the high-temperature regions always correspond to the low gas-density regions and the low-temperature regions always correspond to the high gas-density regions. This is expected because the radiative cooling rate is proportional to the square of the gas density.

The above conclusion can be confirmed in Figure 6. Figure 6 shows the temperature of the IGM as a function of the mass density of the IGM in grid cells at $z=1$ and $z=0$. The density is given in units of the average density of the universe. Only 1 cell out of every 20 has been plotted for clarity. Even though there is scatter, the anticorrelation of the temperature and density can be seen clearly. It also shows that, at present, most of the high-density regions of the IGM have a temperature between $10^{6.5} \mathrm{~K}$ and $10^{7.5} \mathrm{~K}$.

In Figure 7, we have plotted the volume filling factor and mass fraction of the IGM as a function of the temperature at $z=1$ and $z=0$. The plots have been averaged over the four runs. At both epochs, the peak temperature of the mass fraction is lower than that of the volume filling factor, again indicating the high gas-density regions correspond to the low-temperature regions. At $z=1$, most of the gas has a temperature between $3 \times 10^{5} \mathrm{~K}$ and $10^{8} \mathrm{~K}$, with a peak at $\sim 5 \times 10^{6} \mathrm{~K}$. At $z=0$, most of the gas has a temperature between $10^{6} \mathrm{~K}$ and $2 \times 10^{8} \mathrm{~K}$, with a peak at $\sim 10^{7} \mathrm{~K}$. The 


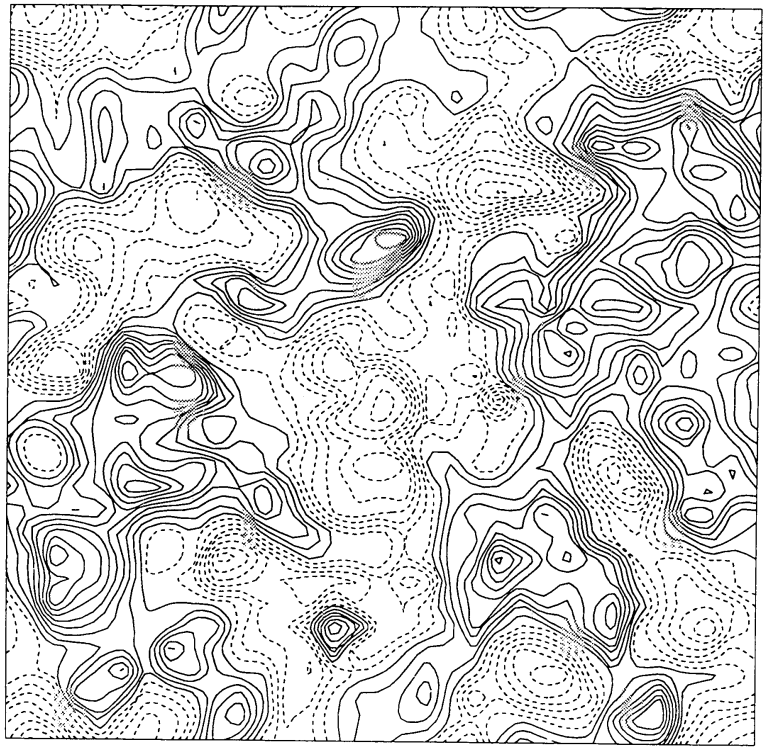

FIG. $5 a$

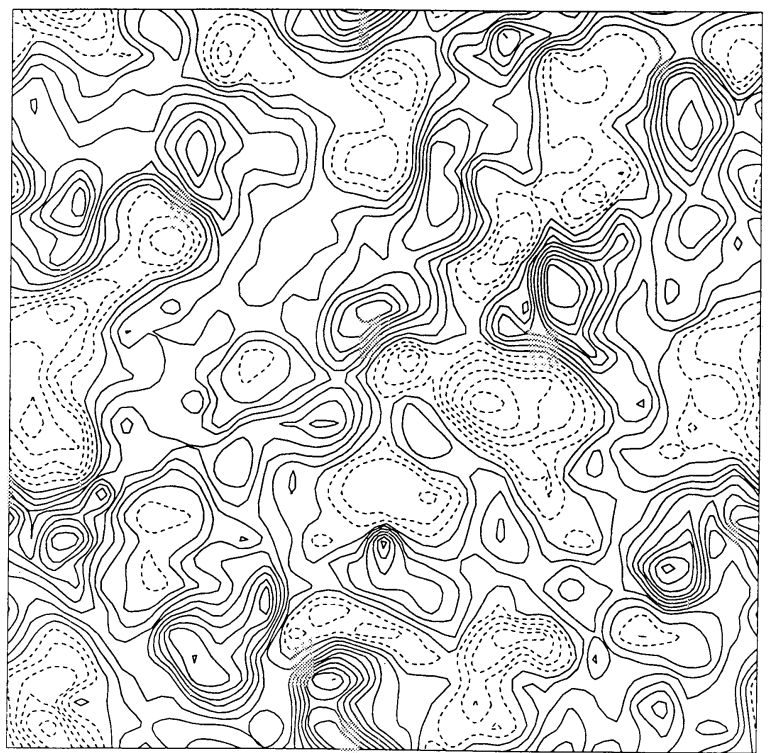

FIG. $5 b$

Fig. 5.-The two-dimensional temperature contour plots of the IGM $(a)$ at $z=1$ and $(b)$ at $z=0$ from the same run as that of Fig. 3. Temperature is the average temperature of the IGM, located between $y=24$ and $y=32$. In $(a)$, the solid lines correspond to regions with the temperature higher than or equal to $10^{7} \mathrm{~K}$, and the dashed lines correspond to regions with the temperature lower than $10^{7} \mathrm{~K}$. In $(b)$, the solid lines correspond to regions with the temperature higher than or equal to $2 \times 10^{7} \mathrm{~K}$, and the dashed lines correspond to regions with the temperature lower than $2 \times 10^{7} \mathrm{~K}$. The contour lines increase in a logarithmic scale with the increment $10^{0.1}$

amount of gas with a temperature below $10^{5} \mathrm{~K}$ is negligible at $z=0$ and $z=1$, indicating that almost all of the hydrogen is ionized since the equilibrium fraction of the neutral hydrogen is only $\sim 10^{-5}$ at the temperature $10^{5} \mathrm{~K}$ (Kang and Shapiro 1990). This agrees with the observational constraint set by Gunn and Peterson (1965). However, the present temperature distribution of the IGM is somewhat sensitive to the adopted

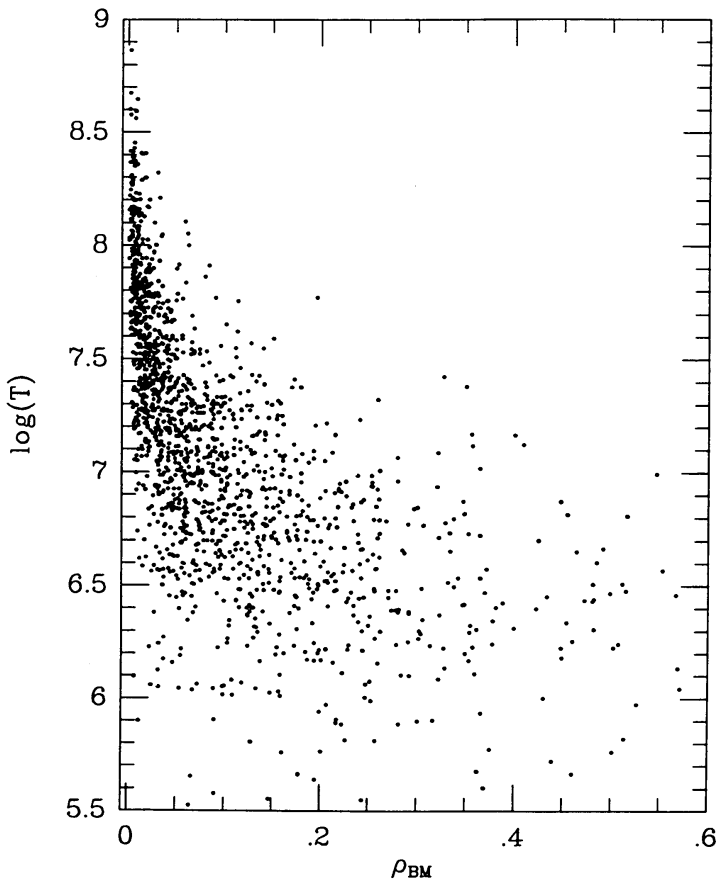

FIG. $6 a$ galaxy formation criteria and the resulting galaxy formation pattern (see Chiang, Ryu, and Vishniac 1989). Hence, the comparison of the calculated temperature distribution with observations may offer a useful constraint on the criteria for galaxy formation.

Figure 8 shows the distribution of density, pressure, and temperature of the IGM along several lines of sight at $z=1$

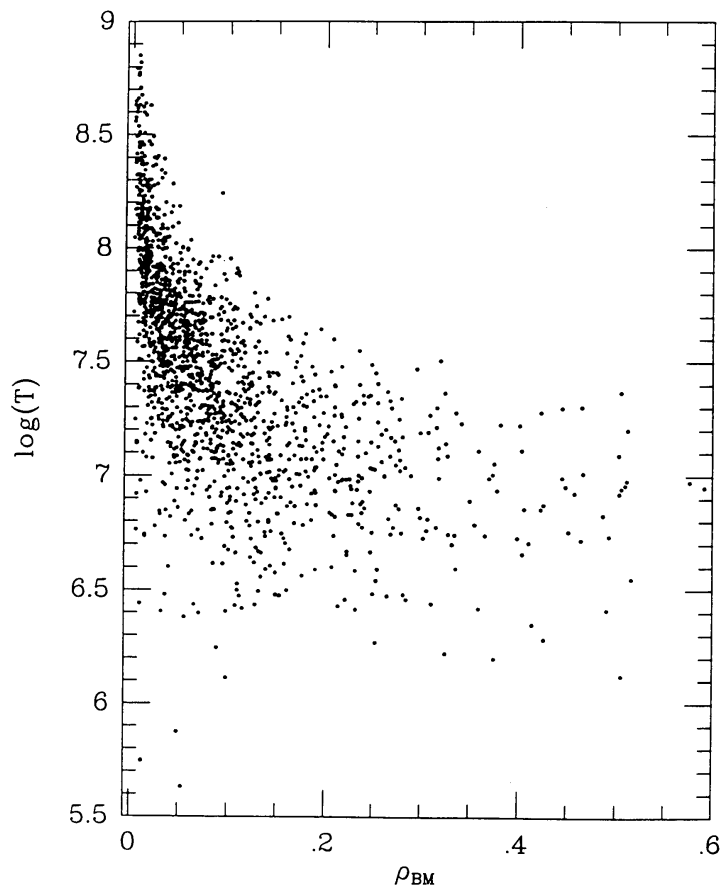

Fig. $6 b$

FIG. 6.-The distribution of the temperature of the IGM in a run as a function of the mass density of the IGM in grid cells $(a)$ at $z=1$ and $(b)$ at $z=0$. The IGM mass density is given in units of the average density of the universe. Only 1 cell out of every 20 has been plotted for clarity. 


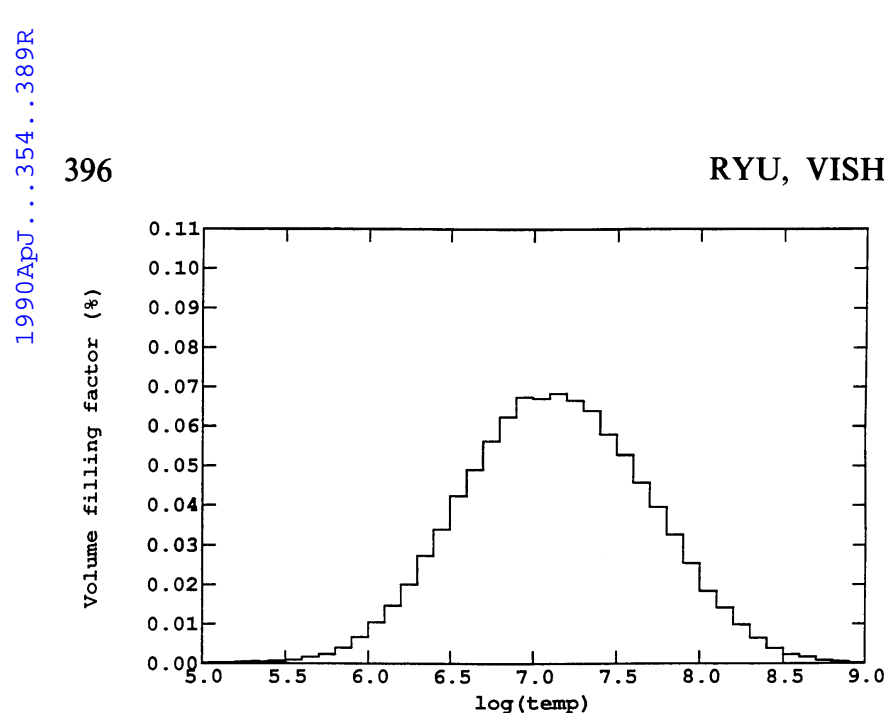

Vol. 354

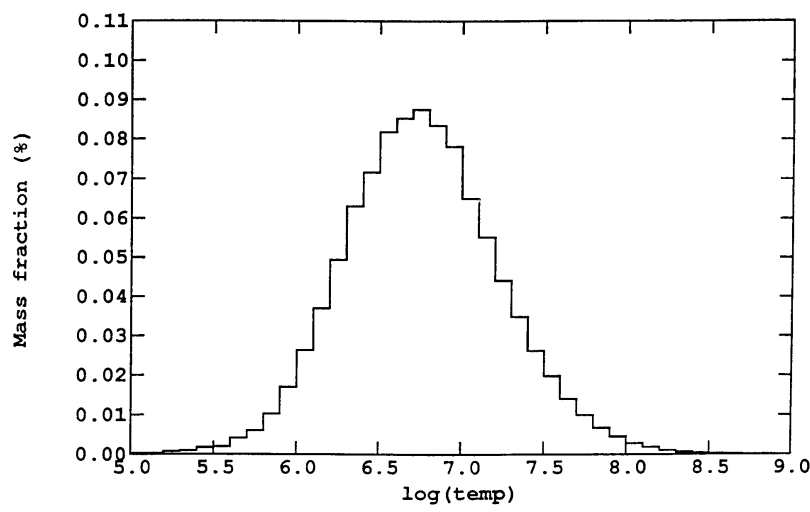

FIG. $7 a$
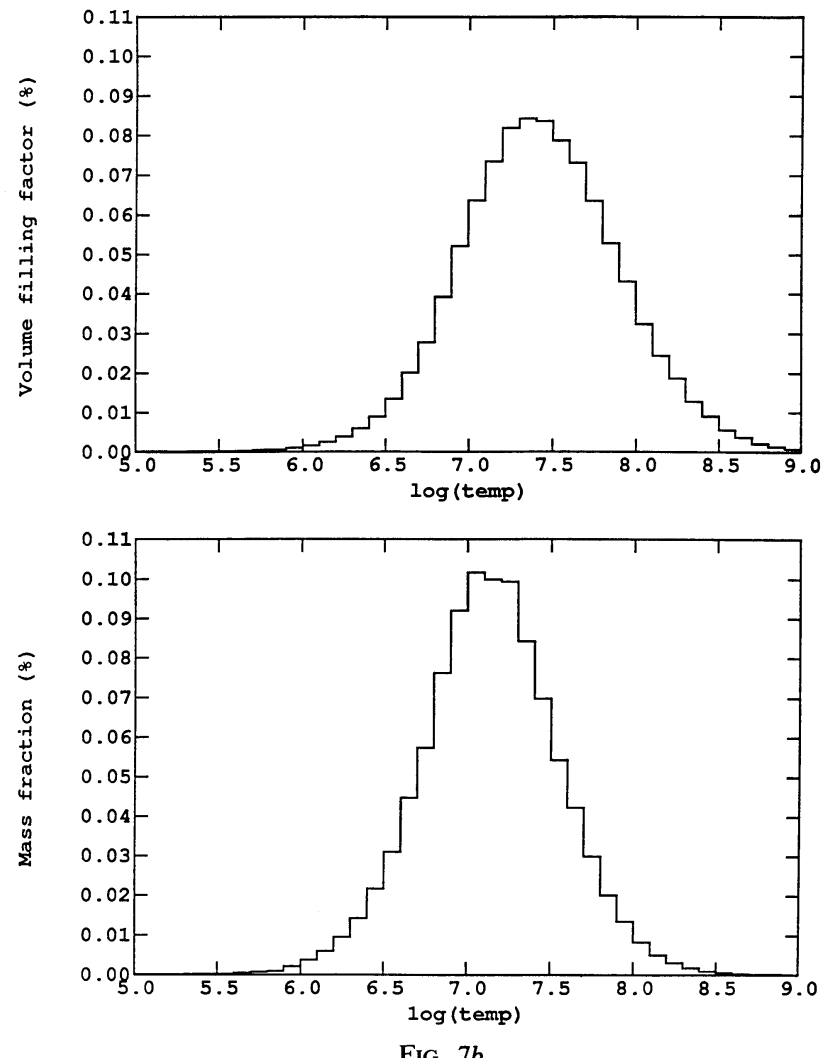

FIG. 7.-The volume filling factor and mass fraction of the IGM as functions of the temperature at $(a) z=1$ and $(b) z=0$. The plots have been averaged over the four runs.

and $z=0$. These quantities have been averaged over $3 \times 3$ cells with twice as much weight on the central cells than on the side cells, and plotted on a logarithmic scale. The solid lines represent the density in units of $10^{-30} \mathrm{~g} \mathrm{~cm}^{-3}$, the long-dashed lines represent the pressure in units of $10^{-17} \mathrm{ergs}^{-3}$, and the short-dashed lines represent to the temperature in units of $10^{2} \mathrm{~K}$. Along the same line of the sight, sometimes the distributions do not change very much from $z=1$ to $z=0$ (see the upper right boxes), but sometimes they change a lot (see the bottom left boxes). As the universe evolves from $z=1$ to $z=0$, the average pressure decreases, even though the average temperature increases. Generally, the pressure is higher in the high-temperature regions. However, the variation in pressure is smaller than those of the density and temperature, even though pressure equilibrium is not achieved. This may be understood from the two following arguments: First, once energy is injected by galaxies, the pressure and temperature remain high in the low-density regions but decrease rapidly in the high-density regions since the cooling rate is proportional to the square of the density. On the other hand, since the density increases further in the high-density regions, there is a greater possibility of galaxy formation and subsequent energy injection which will increase the pressure and temperature. Second, because of the relatively high temperature in all space in our model universe, the sound velocity is expected to be large $\left(C_{s} \sim 10^{3} \mathrm{~km} \mathrm{~s}^{-1}\right.$ at present $)$. Such a large sound velocity can smooth out pressure variations over scales corresponding to several cell width in a time smaller than the age of the universe. The high temperature and, correspondingly, the large sound velocity are partly due to the recent galaxy formation in our model calculations. A different scheme of galaxy formation might result in a different pattern without late galaxy formation and might reduce the tendency toward pressure equilibrium (see Chiang, Ryu, and Vishniac 1989).

\section{e) Fluctuations in the Cosmic Microwave Background Radiation and the Contribution to the $X$-Ray Background Radiation by the IGM}

In the standard big bang cosmology, the cosmic background radiation decouples from the baryonic matter when the temperature drops below $\sim 4000 \mathrm{~K}$. If the universe remains transparent, or the optical depth $\tau$ is small after that recombination epoch, temperature anisotropies in the present cosmic microwave background radiation can be interpreted as the result of inhomogeneities at the recombination epoch (see, for example, Bond and Efstathiou 1984 for detailed calculations). However, if the IGM is reionized by an early generation of the stars or galaxies and the optical depth of the ionized IGM is of order of unity or larger, the original temperature anisotropies will be eliminated. Instead, new temperature fluctuations will be induced by inhomogeneities in the motions of the scattering electrons (see, for example, Ostriker and Vishniac 1986 and Vishniac 1987 for details). Two effects are expected to dominate: the first effect is the Zel'dovich-Sunyaev effect due to the comptonization of photons by a hot electron gas. It shifts the measured temperature at long wavelengths by the amount

$$
\frac{\Delta T}{T}=-2 \int \frac{\sigma_{\mathrm{T}} p_{e}}{m_{e} c^{2}} c d t
$$



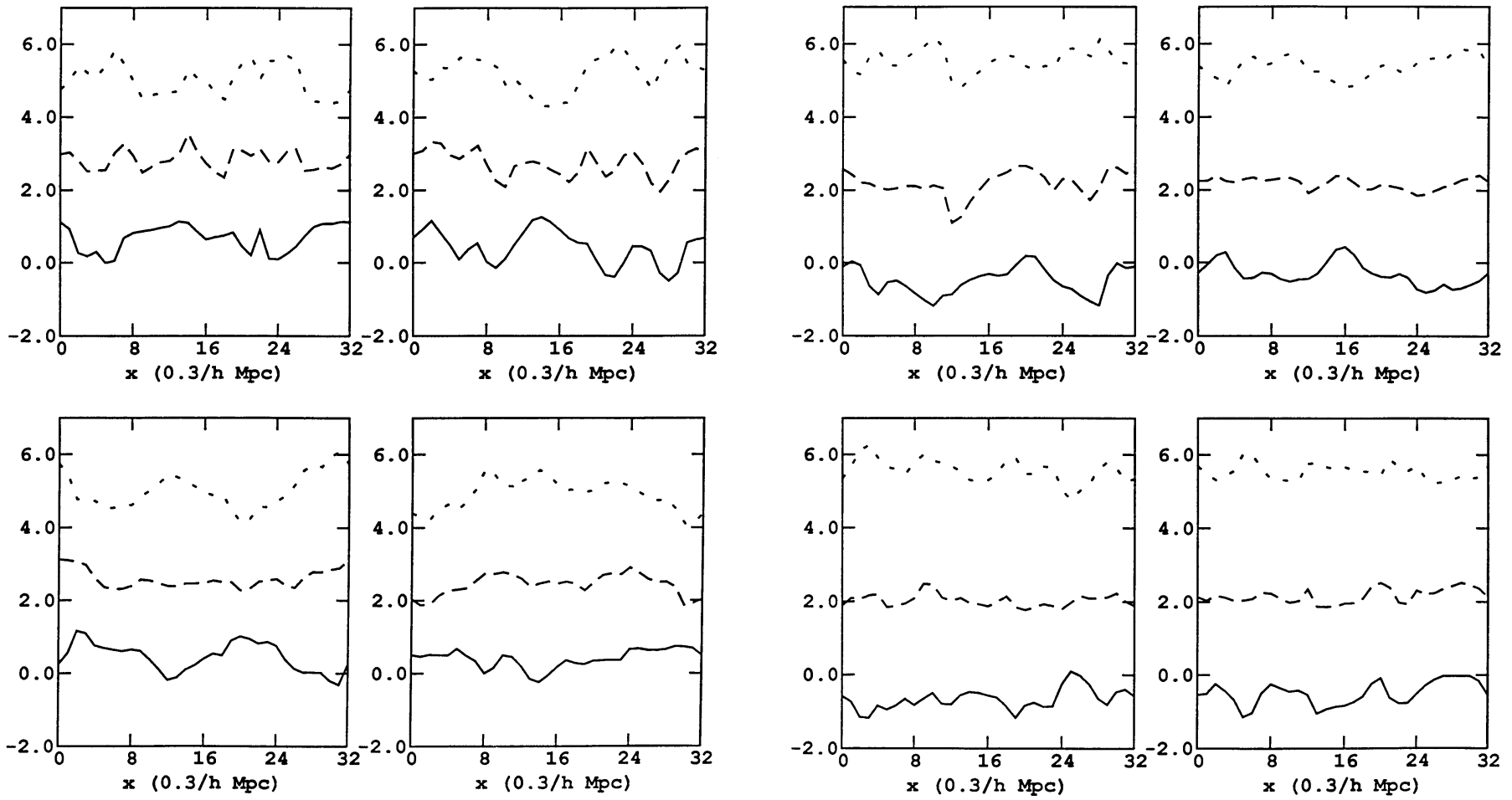

Fig. $8 a$

Fig. $8 b$

FIG. 8. -The profiles of the density, pressure, and temperature of the IGM along four lines of sight at $(a) z=1$ and $(b) z=0$. They have been averaged over nine cells. The solid line represents the density in units of $10^{-30} \mathrm{~g} \mathrm{~cm}^{-3}$, the long-dashed line represents the pressure in units of $10^{-17}$ ergs $\mathrm{cm}^{-3}$, and the short-dashed line represents the temperature in units of $10^{2} \mathrm{~K}$.

(Zel'dovich and Sunyaev 1969). Here, $\sigma_{\mathrm{T}}$ is the Thompson cross section, $p_{e}$ is the electron pressure, and the integral is taken along the path followed by photons on their way to the observer. The second effect is due to the Doppler shift of photons scattered by a cloud of ionized material undergoing bulk motion and distorts the temperature by the amount

$$
\frac{\Delta T}{T}=\int \frac{v_{\|}}{c} n_{e} \sigma_{\mathrm{T}} c d t
$$

(Zel'dovich and Sunyaev 1969). Here, $v_{\|}$is the peculiar velocity parallel to the line of sight and $n_{e}$ is the electron number density.

We have calculated temperature fluctuations in the cosmic microwave background radiation due to the above two effects, by integrating numerically the above equations through the IGM of our model universe. The integrations have been done up to $z=20$ along paths chosen randomly and interpolated properly from the data at $a=1.0,0.5$, and 0.1. Figure 9 shows the distribution of the calculated optical depths and temperature fluctuations. The mean optical depth is $2.72 \times 10^{-2}$ and the standard deviation is $2.27 \times 10^{-3}$. The mean temperature fluctuation is $-9.73 \times 10^{-5}$ and the standard deviation is $4.30 \times 10^{-6}$. A number of interesting results are obtained directly from this calculation. First, in our model universe, the optical depth of the IGM up to the recombination epoch is much smaller than unity. Hence, the total temperature fluctuations in the cosmic microwave background radiation should come from both the inhomogeneities present at the recombination epoch and later inhomogeneities due to the motions of the scattering electrons. Second, the temperature fluctuations induced by the Zel'dovich-Sunyaev effect (the average is equal to $-9.73 \times 10^{-5}$ ) are much larger than those due to the bulk motion of the ionized gas (the average is equal to $1.97 \times 10^{-8}$ ). This is partly because we have included powers only with wavelengths smaller than the computational box size, $9.6 h^{-1} \mathrm{Mpc}$, in our calculations. If power from larger wavelengths is included, the peculiar velocity is expected to increase and the temperature fluctuations induced by the bulk motions are also expected to increase. Third, the standard deviation of the temperature fluctuations is only $\sim 4.30 \times 10^{-6}$, which is about a factor of 3 smaller than the observational limit on temperature anisotropies, $\sim 10^{-5}$. Finally, the distribution of the temperature fluctuations, and also the distribution of optical depths, are lognormal.

Since the first report of its observation by Giaconi et al. (1962), the diffuse X-ray background radiation has been known to exist and wide range of energy bands from a fraction of 1 $\mathrm{keV}$ to at least $100 \mathrm{MeV}$. However, the origin of this radiation still remains controversial. If the diffuse $\mathrm{X}$-ray background radiation in the range of 5-200 keV originates mainly from the thermal bremsstrahlung emission of the hot IGM gas, a temperature $T \sim(4 \pm 1) \times 10^{8}\left(1+z_{\text {emit }}\right) \mathrm{K}$ with a baryonic matter density $\Omega_{\mathrm{BM}} \sim 0.2$ is required (Daly and Turner 1988 and references therein). In our model universe, the average temperature of the IGM gas is only $\sim 10^{7} \mathrm{~K}$ at $z=0$ and $\sim 5 \times 10^{6} \mathrm{~K}$ at $z=1$ and the contribution to the radiation in the range of 5-200 keV by the IGM gas is expected to be negligible. A larger contribution to the softer $\mathrm{X}$-ray radiation is possible, but the spectrum in that energy band is observationally not as well determined. 


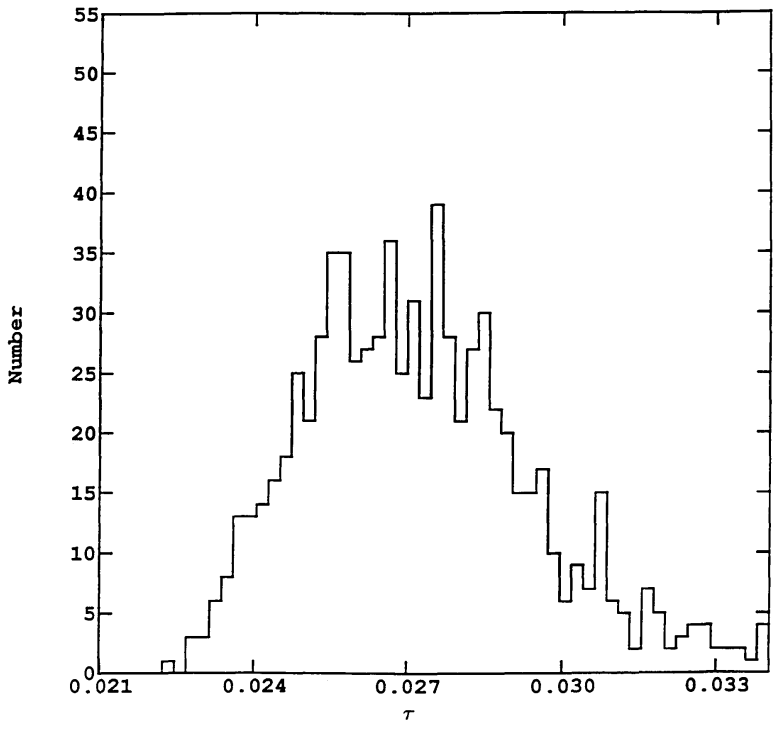

Fig. $9 a$

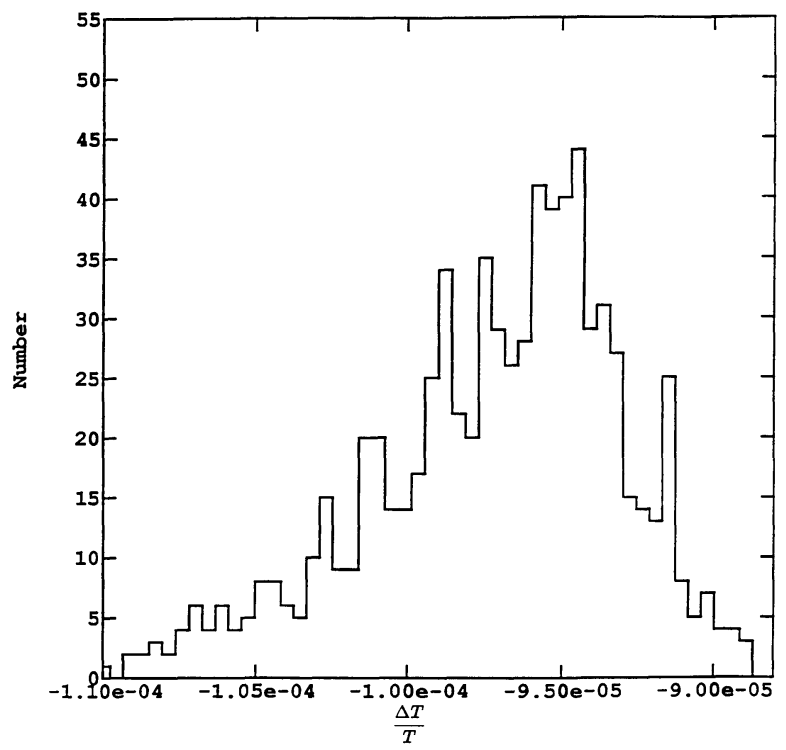

FIG. $9 b$

Fig. 9.-The distribution of $(a)$ the optical depths and $(b)$ the temperature fluctuations in the cosmic microwave background radiation. They have been calculated by integrating up to $z=20$ along the path followed by photons on their way to the observer.

\section{SUMMARY AND CONCLUSIONS}

While most of the previous work on CDM universe models have concentrated only on the evolution of the dark matter distribution and ignored the complicated hydrodynamic evolution of baryonic matter, we have investigated the evolution of the IGM, subject to several assumptions. We have assumed an Einstein-de Sitter universe with $\Omega_{0}=1$ and $h=0.5$ and that $90 \%$ of the matter in the universe is dark matter and $10 \%$ is baryonic matter. The initial conditions have been taken from a constant curvature adiabatic power spectrum. During the model calculations, we have identified galaxies, if some simple criteria were satisfied, and deposited energy into the IGM around the galaxies. Here, galaxy means any object with a significant energy feedback into the IGM. The calculations have been performed with $32^{3}$ dark matter particles and $32^{3}$ cells in a cube with a comoving side length of $L=9.6 h^{-1} \mathrm{Mpc}$.

In our model, there are three parameters that determine when and where galaxies form in the calculations. They are the initial amplitude of the perturbation spectrum, the overdensity required for galaxy formation (actually two numbers), and the size of the region that we test for overdensity. In our calculation we fix the initial amplitude by attempting to match the linear extrapolation of the initial mass fluctuations with the observed galaxy fluctuations. We determine the overdensity by fitting to the current number density of galaxies. Finally, we determine the region size by identifying it with cell size.

Once galaxies form, they subsequently release $10^{60}$ ergs into the IGM over a period of $10^{8} \mathrm{yr}$. The inclusion of substantial energy injection from young galaxies makes our calculations a preliminary test of the possibility of explosive galaxy formation. It is interesting to note that while this energy release seems to substantially effect the evolution of the IGM, it has only a modest effect on the distribution of dark matter. It is possible that a more dramatic effect would have been realized had we set the release energy at $10^{61}$ ergs instead, but there is certainly very little here to indicate that explosive galaxy formation is a necessary part of the CDM model.

Previously, Carlberg (1988) and Chiang, Ryu, and Vishniac
(1989) have considered the baryonic matter in their model calculations of the CDM universe. However, Carlberg did not include the hydrodynamics of the baryonic matter in his calculations. Instead he assumed it was an isothermal gas which was followed with an $\mathrm{N}$-body code. In the paper by Chiang, Ryu, and Vishniac, we used a fluid code to follow the hydrodynamics of the baryons explicitly. However, the gravitational force was calculated by interpolating the initial gravitational potential, and galaxies formed only according to a criterion set to the baryonic matter distribution. Because of the different criteria for galaxy formation, the rate of galaxy formation was very different from that of the present calculations and the subsequent evolution and structure of the IGM were also very different.

The main conclusions from our model are as follows:

1. With our criteria for galaxy formation in equations (2.23) and (2.24), galaxies form from $z \sim 20$ up to present. The pattern of galaxy formation, which depends critically on the criteria for galaxy formation, has important effects on the subsequent evolution of the IGM.

2. The distribution of the IGM is dominated by large connected structures. The volume contained in the high-density region of the IGM does not change very much from $z=1$ to $z=0$.

3. The growth of the power spectrum of the IGM has been suppressed because of the energy released from galaxies and the mass loss from the high-density regions to form galaxies. The two-point correlation function of the IGM also shows a substantial suppression in the late growth of amplitude and indicates the IGM should be less clustered than dark matter.

4. The high-temperature regions of the IGM always correspond to the low-density regions and the low-temperature regions always correspond to the high-density regions, since the equilibrium cooling is proportional to the square of the density. At $z=1$ the IGM has a temperature peak at $\sim 5 \times 10^{6} \mathrm{~K}$, and at $z=0$ the peak is at $\sim 10^{7} \mathrm{~K}$. The fraction of the gas with temperatures below $10^{5} \mathrm{~K}$ is negligible, so that hydrogen is almost completely ionized. The pressure of the 
IGM decreases from $z=1$ to $z=0$. Generally, the pressure is high in the high-temperature region and low in the lowtemperature regions. Pressure variation is usually smaller than those of the density and temperature, even though pressure equilibrium is not achieved. The overall thermal structure of the IGM depends critically on the pattern of the galaxy formation. If we use different criteria for galaxy formation, the thermal structure of the IGM can be quite different.

5. Since the integration of the optical depth up to $z=20$ along the randomly chosen paths is smaller than unity in our model, temperature anisotropies in the cosmic microwave background radiation present at the recombination epoch have not been erased. There are temperature fluctuations induced at late times by inhomogeneities in the motions of scattering electrons. Our calculations give a mean temperature shift at long wavelengths of $-9.73 \times 10^{-5}$ with a standard deviation of $4.30 \times 10^{-6}$. The mean temperature shift is unobservable at present. The standard deviation of the temperature fluctuations is a factor of 3 maller than the observational limits on the temperature anisotropies in the cosmic microwave background radiation at small scales. The amount of the $\mathrm{X}$-ray radiation which can be emitted by the bremsstrahlung of the hot electrons in the IGM is not expected to be significant compared to that of the observed X-ray background radiation.

6. These results depend on our assumptions and the models we have used. Future work with different assumptions and models is desirable.

We are grateful to Hyesung Kang for providing the cooling curve for a primordial plasma and useful discussions. We would also like to thank John M. Scalo, Alexander S. Szalay, Paul R. Shapiro, and J. Craig Wheeler for comments. The calculations have been performed on Cray X-MP/24 at the Center for High Performance Computing of the University of Texas System. This work has been supported in part by NSF grant AST-8451736 and by TARP grant No. 1432 at University of Texas and in part by DOE and by NASA at Fermilab.
Bardeen, J. M., Bond, J. R., Kaiser, N., and Szalay, A. S. 1986, Ap. J., 304, 15

Blumenthal, G. R., Faber, S. M., Primack, J. R., and Rees, M. J. 1984, Nature, 311, 517 .

Bond, J. R., and Efstathiou, G. 1984, Ap. J.(Letters), 285, L45.

Book, D. L., Boris, J. P., and Hain, K. 1975, J. Comput. Phys., 18, 248.

Boris, J. P., and Book, D. L. 1973, J. Comput. Phys., 11, 38. 1976, J. Comput. Phys., 20, 397.

Carlberg, R. G. 1988, Ap. J., 324, 664.

Chiang, W.-H., Ryu, D., and Vishniac, E. T. 1989, Ap. J., 339, 603.

Crotts, A. P. S. 1989, A.J., 336, 550.

Daly, R. A., and Turner, E. L. 1988, preprint.

Davis, M., Efstathiou, G., Frenk, C. S., and White, S. D. M. 1985, Ap. J., 292, 371 .

Efstathiou, G., Davis, M., Frenk, C. S., and White, S. D. M. 1985, Ap. J. Suppl.,

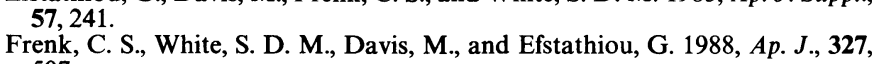
507.

Giacconi, R., Gursky, H., Paolini, F., and Rossi, B., 1962, Phys. Rev. Letters, 9, 439.

Gunn, J. E., and Peterson, B. A. 1965, Ap. J., 142, 1633.

\section{REFERENCES}

Hockney, R. W., and Eastwood, J. W. 1981, Computer Simulations Using Particles (New York: McGraw-Hill).

Kang, H., and Shapiro, P. R. 1990, Ap. J., submitted.

Kurki-Suonio, H., Matzner, R. A., Olive, K. A., and Schramm, D. N. 1989, preprint.

Lynds, C. R. 1971, Ap. J. (Letters), 164, L73.

Ostriker, J. P., Bajtlik, S., and Duncan, R. C. 1988, Ap. J. (Letters), 327, L35.

Ostriker, J. P., and Vishniac, E. T. 1986, Ap. J. (Letters), 306, L51.

Peebles, P. J. E. 1980, The Large-Scale Structure of the Universe (Princeton: Princeton University Press).

. 1982, Ap.J.(Letters), 263, L1.

Sargent, W. L., Young, P. J., Boksenberg, A., and Tytler, D. 1980, Ap. J. Suppl., 42, 41 .

Vishniac, E. T. 1987, Ap. J., 322, 597.

White, S. D. M., Frenk, C. S., Davis, M., and Efstathiou G. 1987, Ap. J., 313, 505 .

Yang, J., Turner, M. S., Steigman, G., Schramm, D. N., and Olive, K. A. 1984, Ap.J., 281, 493 .

Zalesak, S. T. 1979, J. Comput. Phys., 31, 335.

Zel'dovich, Ya. B., and Sunyaev, R. A. 1969, Astr. Ap., 20, 189.

WeI-HWAn ChIANG: IBM Corporation, 33TA/260, Neighborhood Road, Kingston, NY 12401

DongSu RyU: NASA/Fermilab Astrophysics Center, Fermi National Accelerator Laboratory, MS 209, P.O. Box 500, Batavia, IL 60510

EThan T. VishniaC: Department of Astronomy, University of Texas, Austin, TX 78712 